\title{
Chapter 3 \\ The Sensitivity of Population Growth \\ Rate: Three Approaches
}

\subsection{Introduction}

The essence of stable population theory is the fact that a population subject to timeinvariant vital rates will (with a few exceptions not of interest here) converge to a stable structure and grow exponentially at a constant rate (the population growth rate, or intrinsic rate of increase). The calculation of the population growth rate from the vital rates is one of the most important accomplishments of formal demography (Sharpe and Lotka 1911). ${ }^{1}$ Ecologists recognized early on that, by integrating survival and fertility over the life course, the population growth rate provided a powerful tool for describing the population consequences of environmental conditions (e.g., Birch 1953). For the same reason, evolutionary biologists recognized it as a measure of fitness (Fisher 1930), although that concept requires careful consideration of both demographic and genetic processes (Charlesworth 1994; de Vries and Caswell 2018).

This makes the sensitivity analysis of population growth rate an important problem. It has been approached in three ways. The earliest approach (Hamilton 1966) is specific to age-classified models, and relies on differentiation of the characteristic equation. The second (Caswell 1978) applies to stage-classified as well as age-classified models, and uses eigenvalue perturbation theory. The third is based on matrix calculus and is more flexible than its predecessors.

Chapter 3 is modified, under the terms of a Creative Commons Attribution License, from Caswell, H. 2010. Reproductive value, the stable stage distribution, and the sensitivity of the population growth rate to changes in vital rates. Demographic Research 23:531-548, CHal Caswell.

${ }^{1}$ Leonard Euler had obtained the result in 1760, but his derivation rediscovered until 1970 (Keyfitz and Keyfitz 1970). 


\subsection{Hamilton's Equation for Age-Classified Populations}

Consider an age-classified model, in which age $x$ is a continuous variable, with mortality rate $\mu(x)$ and maternity function $m(x)$. The survivorship function is

$$
\ell(x)=\exp \left(-\int_{0}^{x} \mu(a) d a\right)
$$

and the population growth rate $r$ is the solution to the Euler-Lotka equation

$$
1=\int_{0}^{\infty} e^{-r a} \ell(a) m(a) d a .
$$

The stable age distribution, reproductive value function, birth rate, and generation time (mean age of reproduction in the stable population) are given by

$$
\begin{aligned}
c(x) & =\frac{e^{-r x} \ell(x)}{\int_{0}^{\infty} e^{-r a} \ell(a) d a} \quad \text { stable age distribution } \\
v(x) & =\frac{e^{r x}}{\ell(x)} \int_{x}^{\infty} e^{-r a} \ell(a) m(a) d a \quad \text { reproductive value } \\
b & =\left[\int_{0}^{\infty} e^{-r a} \ell(a) d a\right]^{-1} \quad \text { birth rate } \\
\bar{A} & =\int_{0}^{\infty} a e^{-r a} \ell(a) m(a) d a \quad \text { generation time }
\end{aligned}
$$

Sensitivity of $\boldsymbol{r}$ Hamilton (1966) derived the sensitivities of $r$ to changes in mortality and fertility at a specified age $x$. His results are equivalent to

$$
\begin{aligned}
\frac{d r}{d \mu(x)} & =\frac{-c(x) v(x)}{b \bar{A}} \\
\frac{d r}{d m(x)} & =\frac{c(x)}{b \bar{A}}
\end{aligned}
$$

That is, the sensitivity of $r$ to a change in mortality at age $x$ is proportional to the product of the reproductive value at age $x$ and the abundance of age $x$ in the stable age distribution. The sensitivity of $r$ to a change in fertility at age $x$ is proportional to the stable age distribution (and the reproductive value at age 0 , which equals 1 ). The proportionality constant in each case is the inverse of the product of the birth rate and the mean age of reproduction. 
Derivation Hamilton's results are obtained by implicit differentiation of the EulerLotka equation (3.2). We will derive Hamilton's original formulation and then show how it reduces to the relation between the stable age distribution and the reproductive value distribution in (3.7) and (3.8).

First, introduce a perturbation parameter $\theta$ to measure the change in mortality or fertility at the specified age. Writing survival, fertility, and $r$ as functions of $\theta$ gives the Euler-Lotka equation

$$
1=\int_{0}^{\infty} e^{-r(\theta) a} \ell(\theta, a) m(\theta, a) d a .
$$

Differentiating both sides of (3.9) with respect to $\theta$ gives

$$
\begin{aligned}
0= & -\frac{d r(\theta)}{d \theta} \int_{0}^{\infty} a e^{-r(\theta) a} \ell(\theta, a) m(\theta, a) d a \\
& +\int_{0}^{\infty} e^{-r(\theta) a} \frac{d \ell(\theta, a)}{d \theta} m(\theta, a) d a \\
& +\int_{0}^{\infty} e^{-r(\theta) a} \ell(\theta, a) \frac{d m(\theta, a)}{d \theta} d a .
\end{aligned}
$$

Solving (3.10) for $d r / d \theta$ gives

$$
\frac{d r(\theta)}{d \theta}=\frac{1}{\bar{A}}(\underbrace{\int_{0}^{\infty} e^{-r(\theta) a} \frac{d \ell(\theta, a)}{d \theta} m(\theta, a) d a}_{\text {mortality }}+\underbrace{\int_{0}^{\infty} e^{-r(\theta) a} \ell(\theta, a) \frac{d m(\theta, a)}{d \theta} d a}_{\text {fertility }})
$$

Equation (3.11) has two terms, one capturing effects of $\theta$ on mortality and the other capturing effects on fertility.

\subsubsection{Effects of Changes in Mortality}

We want to perturb mortality at one exact age $x$ (remember that age and time are continuous), leaving mortality at all other ages unchanged. To do this, we use the unit impulse function, or Dirac delta function. This is a generalized function defined by

$$
\begin{aligned}
\delta(x) & =0 \quad x \neq 0 \\
\int_{-\infty}^{\infty} \delta(s) d s & =1 .
\end{aligned}
$$


The unit impulse is used in signal processing (e.g., Kamen and Heck 1997, p. 7) to represent the limit of a perturbation of unit strength applied over a shorter and shorter time interval. Think of a normal distribution with mean 0 , in the limit as the variance goes to 0 , while the area under the curve remains at 1 . The most useful properties of the unit impulse, for our application, are

$$
\int_{-\infty}^{\infty} \delta(a-x) f(a) d a=f(x)
$$

and

$$
\int_{-\infty}^{x} \delta(s) d s=H(x)
$$

where $H(x)$ is the Heaviside function, or unit step function, which satisfies $H(x)=$ 0 for $x<0$ and $H(x)=1$ for $x>0$.

We write mortality as

$$
\mu(\theta, a)=\mu(0, a)+\theta \delta(a-x)
$$

where $\delta(x)$ is the unit impulse function. The sensitivity of $r$ to $\mu(x)$ is obtained as the derivative of $r$ with respect to $\theta$, evaluated at $\theta=0$,

$$
\frac{d r}{d \mu(x)}=\left.\frac{d r}{d \theta}\right|_{\theta=0} .
$$

Because only mortality is affected by $\theta$

$$
\begin{aligned}
& \frac{d m(\theta, a)}{d \theta}=0 \\
& \frac{d \mu(\theta, a)}{d \theta}=\delta(a-x) .
\end{aligned}
$$

From (3.1),

$$
\begin{aligned}
\frac{d \ell(\theta, a)}{d \theta} & =-e^{-\int_{0}^{a} \mu(\theta, s) d s} \int_{0}^{a} \delta(a-x) d a \\
& =-\ell(\theta, a) H(a-x) .
\end{aligned}
$$

Substituting into (3.11) and evaluating at $\theta=0$ gives

$$
\frac{d r}{d \mu(x)}=\frac{-1}{\bar{A}}\left(\int_{x}^{\infty} e^{-r a} \ell(a) m(a) d a\right) .
$$


The integral in (3.22) is close to the reproductive value $v(x)$ given by (3.4); specifically,

$$
\int_{x}^{\infty} e^{-r a} \ell(a) m(a) d a=\ell(x) e^{-r x} v(x) .
$$

However, from (3.3) and (3.5), $\ell(x) e^{-r x}=c(x) / b$. Making these substitutions into (3.22) gives the formal relationship (3.7).

\subsubsection{Effects of Changes in Fertility}

Following the same approach, if the perturbation affects fertility at exact age $x$, we write

$$
m(\theta, a)=m(0, a)+\theta \delta(a-x)
$$

Because only fertility is affected by $\theta, d \mu(\theta, a) / d \theta=0$ and $d m(\theta, a) / d \theta=\delta(a-$ $x$ ). Substituting these into (3.11) and evaluating the result at $\theta=0$ gives

$$
\frac{d r}{d m(x)}=\frac{1}{\bar{A}}\left(e^{-r x} \ell(x)\right) .
$$

From (3.3) and (3.5) it can be seen that the numerator is $c(x) / b$, which leads to the formal relationship (3.8).

\subsubsection{History and Perspectives}

Hamilton (1966) obtained the relationship (3.22) in his analysis of the evolution of senescence. From (3.22) and (3.8) it is apparent that (provided $r \geq 0$ ) the magnitudes of the sensitivities of $r$ to mortality and fertility decline with age. These sensitivities measure the selection gradients on age-specific mortality and fertility. Thus Hamilton concluded that the strength of selection against deleterious mutations would necessarily decline with their age of action, that small positive effects at early ages could easily compensate for much larger negative effects at later ages, and that the evolution of senescence was therefore inevitable.

In the years that followed Hamilton's paper, several other authors developed perturbation analysis for $r$, using related methods. Demetrius (1969) used a discrete age-classified model, and Emlen (1970) used Hamilton's results to derive the dynamics of gene frequencies resulting from the selection gradients on age-specific survival and fertility. 
Keyfitz (1971) in a remarkable paper, used implicit differentiation to obtain the sensitivity of population growth rate, life expectancy, birth rates, death rates, and the stable age distribution, apparently independently of Hamilton. He noted the appearance of reproductive value in the sensitivity of $r$ to mortality. Goodman (1971) was apparently the first to note that the sensitivities of $r$ to mortality and fertility could be expressed in terms of the stable age distribution and reproductive value.

When Hamilton's paper appeared, it was regarded as difficult and esoteric, but it had a great impact. It provided the analytical machinery for examining trade-offs between opposing demographic traits, known as antagonistic pleiotropy (Williams 1957; Rose 1991). It also describes the accumulation of deleterious mutations due to the balance between mutation and selection (e.g., Steinsaltz et al. 2005). These ideas are fundamental to the analysis of human aging (e.g., Rose 1991; Wachter and Finch 1997; Carey and Tuljapurkar 2003; Baudisch 2008) and, more generally, the analysis of life history evolution in humans and other species (e.g., Charlesworth 1994; Stearns 1992).

\subsection{Stage-Classified Populations: Eigenvalue Perturbations}

Implicit in Hamilton's analysis is the assumption that the vital rates are functions of age. In many cases, they are not. In humans, characteristics such as education, marital status, health status, or spatial location, may provide important information in addition to age. In other species, the vital rates may depend on developmental stage or body size more than on age. Such populations are described by stageclassified demographic models, of which the age-classified theory is a special case.

Stage-classified demography can be analyzed using matrix population models (Leslie 1945; Caswell 2001). The discrete-time population growth rate $\lambda$ is the dominant eigenvalue of the population projection matrix $\mathbf{A}$ (guaranteed to be real and positive by the Perron-Frobenius theorem). Let $\mathbf{n}(t)$ be the population vector at time $t$, and $\mathbf{A}$ the population projection matrix, with

$$
\mathbf{n}(t+1)=\mathbf{A n}(t)
$$

and the population growth rate is given by the dominant eigenvalue $\lambda$ of $\mathbf{A}$. The stable stage distribution is given by the corresponding right eigenvector $\mathbf{w}$ and the reproductive value function by the left eigenvector $\mathbf{v}$; they satisfy

$$
\begin{aligned}
\mathbf{A w} & =\lambda \mathbf{w} \\
\mathbf{v}^{\top} \mathbf{A} & =\lambda \mathbf{v}^{\top}
\end{aligned}
$$


Sensitivity of $\lambda$ The effects of perturbations on population growth are approached by looking for the sensitivity of an eigenvalue to changes in the entries of a matrix. We will see that the sensitivity of $\lambda$ to a change in the entry $a_{i j}$ of $\mathbf{A}$ is (Caswell 1978)

$$
\frac{\partial \lambda}{\partial a_{i j}}=\frac{v_{i} w_{j}}{\mathbf{v}^{\top} \mathbf{w}} .
$$

The entry $a_{i j}$ measures the per-capita production of stage $i$ by stage $j$. Thus the effect of a change in $a_{i j}$ is proportional to the reproductive value of the destination stage and to the abundance of the origin stage in the stable population. This is a generalization of the relationships (3.7) and (3.8) obtained from Hamilton's analysis.

Derivation The eigenvalue $\lambda$ is a solution to the characteristic equation of $\mathbf{A}$, which generalizes the Euler-Lotka equation (3.2). Except in special cases, however, the characteristic equation cannot be written down explicitly, making the implicit differentiation approach used by Hamilton impossible. Instead, the relationship (3.29) is obtained by a perturbation expansion. Suppose that $\mathbf{A}$ is perturbed to $\mathbf{A}+\Delta \mathbf{A}$. This will result in perturbations of $\lambda$ and of $\mathbf{w}$, which must satisfy

$$
(\mathbf{A}+\Delta \mathbf{A})(\mathbf{w}+\Delta \mathbf{w})=(\lambda+\Delta \lambda)(\mathbf{w}+\Delta \mathbf{w}) .
$$

Expanding the products, setting second order terms to zero, and remembering that $\mathbf{A w}=\lambda \mathbf{w}$ gives

$$
\mathbf{A}(\Delta \mathbf{w})+(\Delta \mathbf{A}) \mathbf{w}=\lambda(\Delta \mathbf{w})+(\Delta \lambda) \mathbf{w}
$$

Multiply on the left by $\mathbf{v}^{\top}$ and simplify to obtain

$$
(\Delta \lambda) \mathbf{v}^{\top} \mathbf{w}=\mathbf{v}^{\top}(\Delta \mathbf{A}) \mathbf{w} .
$$

If the perturbation affects only one entry, say $a_{i j}$, of $\mathbf{A}$, then

$$
\Delta \lambda=\frac{v_{i} w_{j}\left(\Delta a_{i j}\right)}{\mathbf{v}^{\top} \mathbf{w}} .
$$

Dividing both sides by $\Delta a_{i j}$ and taking the limit as $\Delta a_{i j} \rightarrow 0$ gives the sensitivity result (3.29).

\subsubsection{Age-Classified Models as a Special Case}

To compare (3.29) with Hamilton's results (3.7) and (3.8), consider an age-classified matrix (a Leslie matrix) with fertilities $F_{i}$ in the first row, survival probabilities $P_{i}$ on 
the subdiagonal, and zeros elsewhere (Leslie 1945; Keyfitz 1968). In this case (3.29) simplifies to

$$
\begin{aligned}
\frac{\partial \lambda}{\partial P_{i}} & =\frac{v_{i+1} w_{i}}{\mathbf{v}^{\top} \mathbf{w}} \\
\frac{\partial \lambda}{\partial F_{i}} & =\frac{v_{1} w_{i}}{\mathbf{v}^{\top} \mathbf{w}} .
\end{aligned}
$$

Equation (3.34) corresponds to (3.7); the sensitivity is proportional to the product of the reproductive value and the stable stage distribution. Equation (3.35) corresponds to (3.8), and shows why reproductive value is apparently missing from (3.8): reproductive value at birth $[v(0)$ in Hamilton's notation] is scaled to equal 1.

\subsubsection{Sensitivity to Lower-Level Demographic Parameters}

The entries of $\mathbf{A}$ are often functions of other, lower-level parameters. The sensitivity of $\lambda$ to these parameters is obtained by the chain rule. For example, suppose that stage 1 may contribute individuals to stages 2 or 3 (Fig. 3.1). Write the transition probabilities as

$$
\begin{aligned}
& a_{21}=\gamma \sigma \\
& a_{31}=(1-\gamma) \sigma
\end{aligned}
$$

where $\sigma$ is the survival probability and $\gamma$ the probability that the individual moves to stage 2 , conditional on survival. Then the sensitivities of $\lambda$ to $\gamma$ and to $\sigma$ are given by

$$
\begin{aligned}
\frac{d \lambda}{d \sigma} & =\frac{\partial \lambda}{\partial a_{21}} \frac{d a_{21}}{d \sigma}+\frac{\partial \lambda}{\partial a_{31}} \frac{d a_{31}}{d \sigma} \\
& =\frac{w_{1}\left[\gamma v_{2}+(1-\gamma) v_{3}\right]}{\mathbf{v}^{\top} \mathbf{w}}
\end{aligned}
$$

Fig. 3.1 An example of lower-level parameters appearing in a portion of a life cycle. Individuals in stage 1 survive with probability $\sigma$, and, conditional on survival, move to stage 2 with probability $\gamma$ and to stage 3 with probability $1-\gamma$

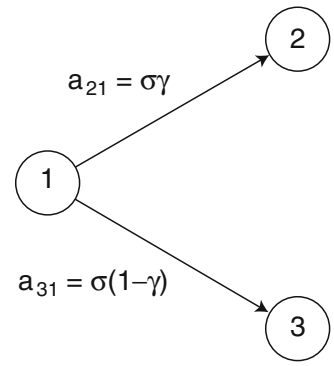




$$
\begin{aligned}
\frac{d \lambda}{d \gamma} & =\frac{\partial \lambda}{\partial a_{21}} \frac{d a_{21}}{d \gamma}+\frac{\partial \lambda}{\partial a_{31}} \frac{d a_{31}}{d \gamma} \\
& =\frac{\sigma w_{1}\left(v_{2}-v_{3}\right)}{\mathbf{v}^{\top} \mathbf{w}} .
\end{aligned}
$$

The sensitivity to survival is proportional to the weighted average of the reproductive values of the destination stages, and the sensitivity to the transition probability $\gamma$ is proportional to the difference in reproductive value between the destination stages.

\subsubsection{History}

I first encountered the basis for this perturbation expansion in a paper by C.A. Desoer in the proceedings of an engineering conference (Desoer 1967). ${ }^{2}$ Eigenvalue perturbations were of particular interest to engineers in the 1960s as part of a shift from frequency-domain methods to state-space methods in the study of linear systems (Zadeh and Desoer 1963). However, the result dates back to Jacobi (1846), and has been independently rediscovered many times (e.g., Faddeev 1959; Papoulis 1966; Franklin 1968). In population biology, this perturbation approach has been extended to many other sensitivity problems, including the sensitivity of subdominant eigenvalues and transient behavior, of growth rates in periodic and stochastic environments, of the eigenvectors, and of the spreading speed in biological or demographic invasions (see Caswell (2001) for reviews and references).

\subsection{Growth Rate Sensitivity via Matrix Calculus}

Matrix calculus provides a still more general approach to the sensitivity analysis of the population growth rate. Equation (3.29) perturbs only a single entry of $\mathbf{A}$; derivatives with respect to other parameters are assembled by summing their effects over all the entries of $\mathbf{A}$, as in (3.41). Using matrix calculus, we now consider $\lambda$ as a scalar function of $\mathbf{A}$ and $\mathbf{A}$ as a matrix-valued function of a parameter vector $\boldsymbol{\theta}$.

Sensitivity of $\lambda$ We will show that the derivative of $\lambda$ with respect to $\boldsymbol{\theta}$ is

$$
\frac{d \lambda}{d \boldsymbol{\theta}^{\top}}=\left(\frac{\mathbf{w}^{\top} \otimes \mathbf{v}^{\top}}{\mathbf{v}^{\top} \mathbf{w}}\right)\left(\frac{d \operatorname{vec} \mathbf{A}}{d \boldsymbol{\theta}^{\top}}\right),
$$

\footnotetext{
${ }^{2} \mathrm{By}$ a fortunate accident; I was searching for something completely different. We may wonder whether the chances of such coincidences are higher or lower in the internet search era.
} 
where $\otimes$ denotes the Kronecker product. If $\boldsymbol{\theta}$ is a $p \times 1$ vector of parameters, then $d \lambda / d \boldsymbol{\theta}^{\top}$ is a $1 \times p$ matrix whose $i$ th entry is $d \lambda / d \theta_{i}$.

Derivation Following the steps in Chap. 2, begin by taking the differential of both sides of (3.27) to give

$$
(d \mathbf{A}) \mathbf{w}+\mathbf{A}(d \mathbf{w})=(d \lambda) \mathbf{w}+\lambda(d \mathbf{w}) .
$$

Multiply both sides on the left by $\mathbf{v}^{\top}$ and simplify to obtain

$$
(d \lambda) \mathbf{v}^{\top} \mathbf{w}=\mathbf{v}^{\top}(d \mathbf{A}) \mathbf{w}
$$

Next, apply the vec operator to both sides of (3.44). Since the left side is a scalar, the vec operator has no effect. The right side is a product of three quantities, so Roth's theorem implies that

$$
(d \lambda) \mathbf{v}^{\top} \mathbf{w}=\left(\mathbf{w}^{\top} \otimes \mathbf{v}^{\top}\right) d \operatorname{vec} \mathbf{A} .
$$

The First Identification Theorem then gives

$$
\frac{d \lambda}{d \operatorname{vec}^{\top} \mathbf{A}}=\frac{\mathbf{w}^{\top} \otimes \mathbf{v}^{\top}}{\mathbf{v}^{\top} \mathbf{w}} .
$$

Finally, the chain rule (2.18) gives us

$$
\frac{d \lambda}{d \boldsymbol{\theta}^{\top}}=\frac{d \lambda}{d \operatorname{vec}^{\top} \mathbf{A}} \frac{d \operatorname{vec} \mathbf{A}}{d \boldsymbol{\theta}^{\top}} .
$$

The matrix calculus approach is particularly powerful because of the flexibility in specifying the effect of $\boldsymbol{\theta}$ on the vital rates. Suppose that $\mathbf{A}$ depends on a vector $\boldsymbol{\sigma}$ of survival probabilities, which are a function of the concentration $X$ of a pollutant, which in turn is changing as a function of time $t$. The rate of change of $\lambda$ over time is

$$
\frac{d \lambda}{d t}=\left(\frac{d \lambda}{d \operatorname{vec}^{\top} \mathbf{A}}\right)\left(\frac{d \operatorname{vec} \mathbf{A}}{d \boldsymbol{\sigma}^{\top}}\right)\left(\frac{d \boldsymbol{\sigma}}{d X}\right)\left(\frac{d X}{d t}\right)
$$

Each of the terms in (3.48) can be evaluated separately; the matrix product gives the correct dimension for the final sensitivity result (a $1 \times 1$ scalar in this case).

\subsection{Second Derivatives of Population Growth Rate}

The second derivatives of $\lambda$ measure the curvature of the response to changes in parameters. They have important applications in evolutionary demography, where they indicate the action of stabilizing, disruptive, or correlational selection on 
fitness-related traits (e.g., Phillips and Arnold 1989; Caswell 2001), in adaptive dynamics, where they help determine the stability of evolutionary singular strategies (e.g., Diekmann 2004), and in extending sensitivity analysis to second-order effects.

Since the first derivatives of $\lambda$ are written, in Eqs. (3.29) and (3.46), in terms of the right and left eigenvectors of $\mathbf{A}$, the second derivatives of $\lambda$ require the first derivatives of those eigenvectors. Caswell (1996) derived the second derivatives of $\lambda$ to entries of $\mathbf{A}$ by an extension of the method in Sect. 3.3. However, a more general and rigorous method is available using matrix calculus.

Consider a (scalar) variable $\xi$ which is a function of a vector $\boldsymbol{\theta}$ of parameters. The complete set of second derivatives of $\xi$ are given by the Hessian matrix

$$
\mathbf{H}=\left(\frac{\partial^{2} \xi}{\partial \theta_{i} \partial \theta_{j}}\right)
$$

Magnus and Neudecker (1988) proved (their Second Identification Theorem) that if the second differential of $\xi$ can be written as

$$
d^{2} \xi=d \boldsymbol{\theta}^{\top} \mathbf{B} d \boldsymbol{\theta}
$$

for some matrix $\mathbf{B}$, then

$$
\mathbf{H}=\frac{1}{2}\left(\mathbf{B}+\mathbf{B}^{T}\right) .
$$

Shyu and Caswell (2014) used this approach to derive the second derivatives of the population growth rate $\lambda$, the continuous-time population growth rate $r=\log \lambda$, and the net reproductive rate $R_{0}$, to changes in either the entries of $\mathbf{A}$ or to arbitrary lower-level parameters of which $\mathbf{A}$ is a function. We will not explore second derivatives in this book, but Shyu's other work (Shyu and Caswell 2016a,b) applies them to analyze the evolutionary demography of sex ratios, and Caswell and Shyu (2017) use them to analyze the effects of mortality on the selection gradients on senescence.

\subsection{Conclusion}

Each of the three approaches to growth rate sensitivity, leading to Eqs. (3.7), (3.8), (3.29), and (3.42), uses its own analytical methods. They agree, however, in showing how the sensitivity of population growth rate can be written in terms of the stable stage distribution and the reproductive value. In general, the effect of a change in the rate at which individuals move from stage $j$ to stage $i$ is proportional to the abundance of the origin stage $(j)$ and the reproductive value of the destination stage $(i)$. If a transition yields individuals with low reproductive value, or if few individuals are available to experience the change in the rate of transition, the effect on population growth will be small. 


\section{Bibliography}

Baudisch, A. 2008. Inevitable aging? Contributions to evolutionary-demographic theory. SpringerVerlag, Berlin, Germany.

Birch, L. C. 1953. Experimental background to the study of the distribution and abundance of insects: I. The influence of temperature, moisture and food on the innate capacity for increase of three grain beetles. Ecology 34:698-711.

Carey, J. R., and S. Tuljapurkar, editors, 2003. Life span: evolutionary, ecological, and demographic perspectives, volume 29. Population Council, New York, New York.

Caswell, H. 1978. A general formula for the sensitivity of population growth rate to changes in life history parameters. Theoretical Population Biology 14:215-230.

Caswell, H. 1996. Second derivatives of population growth rate: calculation and applications. Ecology 77:870-879.

Caswell, H. 2001. Matrix Population Models: Construction, Analysis, and Interpretation. 2nd edition. Sinauer Associates, Sunderland, MA.

Caswell, H., and E. Shyu, 2017. Senescence, selection gradients, and mortality. Chapter 4, pages 56-82 in R. P. Shefferson, O. R. Jones, and R. Salguero-Gómez, editors. The Evolution of Senescence in the Tree of Life. Cambridge University Press.

Charlesworth, B. 1994. Evolution in Age-Structured Populations. 2nd edition. Cambridge University Press, Cambridge, UK.

de Vries, C., and H. Caswell. 2018. Stage-stuctured evolutionary demography: linking life histories, population genetics, and ecological dynamics. (in prep).

Demetrius, L. 1969. The sensitivity of population growth rate to perturbations in the life cycle components. Mathematical Biosciences 4:129-136.

Desoer, C. A., 1967. Perturbations of eigenvalues and eigenvectors of a network. Pages 8-11 in Fifth Annual Allerton Conference on Circuit and System Theory. University of Illinois, Urbana, Illinois, USA.

Diekmann, O. 2004. A beginner's guide to adaptive dynamics. Banach Center Publications 63:4786.

Emlen, J. M. 1970. Age specificity and ecological theory. Ecology 51:588-601.

Faddeev, D. K. 1959. The conditionality of matrices ("Ob obuslovlennosti matrits"). Matematicheskii institut Steklov, Trudy No 53:387-391.

Fisher, R. A. 1930. The Genetical Theory of Natural Selection. Revised 1956, Dover Publications edition. Oxford University Press, Oxford, U.K.

Franklin, J. N. 1968. Matrix theory. Prentice-Hall, Englewood Cliffs, New Jersey, USA.

Goodman, L. A. 1971. On the sensitivity of the intrinsic growth rate to changes in the age-specific birth and death rates. Theoretical Population Biology 2:339-354.

Hamilton, W. D. 1966. The moulding of senescence by natural selection. Journal of Theoretical Biology 12:12-45.

Jacobi, C. J. G. 1846. Uber ein leichtes Verfagren die in der Theorie der Sacularstorungen Vorkommenden Gleichungen numerisch aufsulosen. J. Reine Angew. Math 30:51-95.

Kamen, E. W., and B. S. Heck. 1997. Fundamentals of signals and systems. Prentice Hall, Upper Saddle River, New Jersey, USA.

Keyfitz, N. 1968. Introduction to the Mathematics of Population. Addison-Wesley, Reading, Massachusetts, USA.

Keyfitz, N. 1971. Linkages of intrinsic to age-specific rates. Journal of the American Statistical Association 66:275-281.

Keyfitz, N., and B. Keyfitz. 1970. Euler, L. 1760. Recherches générales sur la mortalité et la multiplication [General researches on mortality and multiplication]. Mémoires de l'Académie Royale des Sciences et Belles Lettres 16:144-164. Translated by N. Keyfitz and B. Keyfitz. Theoretical Population Biology 1:307-314.

Leslie, P. H. 1945. On the use of matrices in certain population mathematics. Biometrika 33:183212. 
Magnus, J. R., and H. Neudecker. 1988. Matrix differential calculus with applications in statistics and econometrics. John Wiley and Sons, New York, New York.

Papoulis, A. 1966. Perturbations of the natural frequencies and eigenvectors of a network. IEEE Transactions on Circuit Theory CT-13:188-195.

Phillips, P. C., and S. J. Arnold. 1989. Visualizing multivariate selection. Evolution 43:1209-1222.

Rose, M. R. 1991. Evolutionary biology of aging. Oxford University Press, Oxford, U.K.

Sharpe, F. R., and A. J. Lotka. 1911. A problem in age-distribution. Philosophical Magazine 21:435-438.

Shyu, E., and H. Caswell. 2014. Calculating second derivatives of population growth rates for ecology and evolution. Methods in Ecology and Evolution 5:473-482.

Shyu, E., and H. Caswell. 2016a. A demographic model for sex ratio evolution and the effects of sex-biased offspring costs. Ecology and Evolution 6:1470-1492.

Shyu, E., and H. Caswell. 2016b. Frequency-dependent two-sex models: a new approach to sex ratio evolution with multiple maternal conditions. Ecology and Evolution 6:6855-6879.

Stearns, S. C. 1992. The evolution of life histories. Oxford University Press, Oxford, England.

Steinsaltz, D., S. N. Evans, and K. W. Wachter. 2005. A generalized model of mutation-selection balance with applications to aging. Advances in Applied Mathematics 35:16-33.

Wachter, K. W., and C. E. Finch. 1997. Between Zeus and the salmon: the biodemography of longevity. National Academy Press, Washington DC, USA.

Williams, G. C. 1957. Pleiotropy, natural selection, and the evolution of senescence. Evolution 11:398-411.

Zadeh, L. A., and C. A. Desoer. 1963. Linear system theory. McGraw-Hill, New York, New York, USA.

Open Access This chapter is licensed under the terms of the Creative Commons Attribution 4.0 International License (http://creativecommons.org/licenses/by/4.0/), which permits use, sharing, adaptation, distribution and reproduction in any medium or format, as long as you give appropriate credit to the original author(s) and the source, provide a link to the Creative Commons licence and indicate if changes were made.

The images or other third party material in this chapter are included in the chapter's Creative Commons licence, unless indicated otherwise in a credit line to the material. If material is not included in the chapter's Creative Commons licence and your intended use is not permitted by statutory regulation or exceeds the permitted use, you will need to obtain permission directly from the copyright holder. 